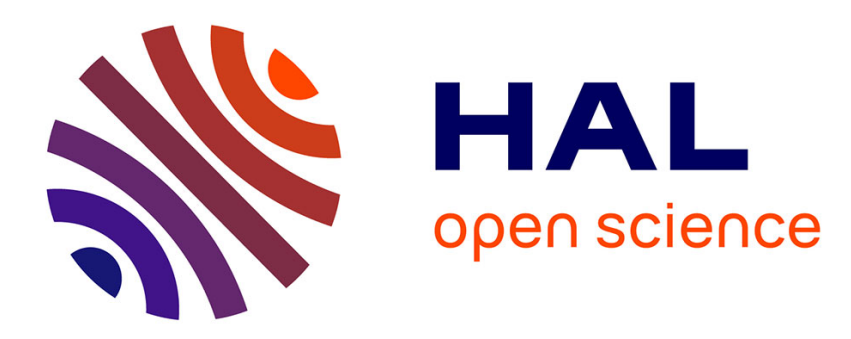

\title{
L'utilisation d'un mélange de sels fondus pour le stockage de chaleur
}

\author{
M. Roche
}

\section{To cite this version:}

M. Roche. L'utilisation d'un mélange de sels fondus pour le stockage de chaleur. Revue de Physique Appliquée, 1980, 15 (4), pp.895-902. 10.1051/rphysap:01980001504089500 . jpa-00244802

\section{HAL Id: jpa-00244802 https://hal.science/jpa-00244802}

Submitted on 1 Jan 1980

HAL is a multi-disciplinary open access archive for the deposit and dissemination of scientific research documents, whether they are published or not. The documents may come from teaching and research institutions in France or abroad, or from public or private research centers.
L'archive ouverte pluridisciplinaire HAL, est destinée au dépôt et à la diffusion de documents scientifiques de niveau recherche, publiés ou non, émanant des établissements d'enseignement et de recherche français ou étrangers, des laboratoires publics ou privés. 


\title{
L'utilisation d'un mélange de sels fondus pour le stockage de chaleur
}

\author{
M. Roche \\ E.D.F., Département Systèmes énergétiques, 6 quai Watier, 78400 Chatou, France
}

(Reçu le 19 octobre 1979, révisé le 31 décembre 1979, accepté le 7 janvier 1980)

\begin{abstract}
Résumé. - L'exploitation rationnelle de l'énergie solaire nécessite le stockage d'une partie de la chaleur produite aux heures d'ensoleillement pour pouvoir la restituer aux heures de consommation, ou tout au moins pour assurer la continuité du fonctionnement pendant les passages nuageux. Les sels fondus et en particulier le H.T.S. (Heat Transfer Salt) apparaissent comme les meilleurs fluides de stockage thermique. En effet, ils permettent un stockage d'une grande densité pour un faible coût et bénéficient de près de 50 ans d'expérience industrielle. Après un rappel des propriétés essentielles de ces milieux nous aborderons les problèmes spécifiques du H.T.S. : dégradation, corrosion, technologie et sécurité.
\end{abstract}

\begin{abstract}
Rational use of solar energy needs to store some part of the heat produced at the sunny hours in order to restitute it during utilization hours or at least to ensure continuous working during passages of clouds. Molten salts and specially H.T.S. (Heat Transfer Salt) look like the best thermal storage fluids. They allow high density storage at a low cost and they have been used for fifty years in industrial processes. After reminding essential properties of these fluids we shall approach specific problems of H.T.S. : stability, corrosion, technology and safety.
\end{abstract}

1. Introduction. - Afin d'utiliser l'énergie solaire avec un maximum d'efficacité, il est souhaitable de pouvoir la stocker et s'en servir aux heures de grande consommation. Dans une centrale électrosolaire, on recueille d'abord l'énergie sous forme de chaleur puis on la transforme en énergie mécanique par l'intermédiaire d'une turbine à vapeur et finalement cette énergie mécanique entraîne un alternateur qui produit de l'électricité. Le stockage de l'énergie sous sa forme première, c'est-à-dire sous forme de chaleur semble le plus facile à réaliser à condition que l'on trouve un support pour cette chaleur. Ce support sera un corps facile à manipuler, donc liquide de préférence, et utilisable entre $250^{\circ} \mathrm{C}$ et $450^{\circ} \mathrm{C}$ au moins, de façon à entraîner une turbine à vapeur de quelques mégawatts avec le meilleur rendement. Comme il sera présent en quantités importantes (plusieurs centaines de tonnes) il est important que ce produit soit peu coûteux et pose un minimum de problèmes de sécurité.

Ces différentes exigences ont conduit pour la centrale THEMIS à choisir un fluide de stockage qui est un mélange de sels fondus et dont les applications industrielles sont déjà nombreuses (références [1 et 2]). Nous allons exposer les raisons de ce choix en présentant les principales propriétés de ce fluide ainsi que les problèmes qui pourraient être soulevés par son utilisation.
2. Les sels de transfert thermique. - Les mélanges de sels fondus auxquels nous nous intéressons sont obtenus à partir de 3 produits de base : le nitrate de sodium $\mathrm{NaNO}_{3}$, le nitrate de potassium $\mathrm{KNO}_{3}$ et le nitrite de sodium $\mathrm{NaNO}_{2}$ qui présentent les avantages d'être peu coûteux et peu corrosifs. Pris séparément ils ne sont liquides qu'au-dessus de $300^{\circ} \mathrm{C}$ mais on peut réaliser des mélanges eutectiques qui fondent à des températures bien plus basses. Le mélange le plus couramment utilisé, dont nous donnons la composition, est usuellement nommé H.T.S. (Heat Transfer Salt) ou encore HITEC (dénomination de la Sté Dupont de Nemours).

Tableau I. - Composition du H.T.S.

\begin{tabular}{cc}
$\begin{array}{c}\text { Pourcentage } \\
\text { en masse }\end{array}$ & $\begin{array}{c}\text { Pourcentage } \\
\text { en moles }\end{array}$ \\
\hline 53 & - \\
40 & 44 \\
7 & 49 \\
\end{tabular}

L'usage de ce fluide qui fond à $146^{\circ} \mathrm{C}$ et peut atteindre $500{ }^{\circ} \mathrm{C}$ est largement répandu dans l'industrie chimique pour maintenir à la température optimale divers réacteurs. 
On l'utilise également en métallurgie pour les traitements thermiques (revenu ou trempe des aciers) ou pour les traitements de surface anticorrosion (on utilise alors les propriétés oxydantes des nitrates et des nitrites pour former une mince couche d'oxyde protecteur).

On dispose donc d'une certaine expérience dans l'utilisation des sels fondus, mais dans le cas d'une centrale solaire il faudra utiliser une quantité inhabituelle de ce fluide, aussi plusieurs séries d'essais ont été faites dans le but de mieux connaître la température maximale d'utilisation (problèmes de dégradation et de corrosion) et la technologie à utiliser. En particulier une boucle d'essais a été construite sur le site E.D.F. de Chatou, à la division T.E.N., avec les caractéristiques suivantes [3] :

- 2 tonnes de sel en circulation;

- chaudière électrique de $140 \mathrm{~kW}_{\text {th }}$ permettant de porter le fluide de $250^{\circ} \mathrm{C}$ à $500^{\circ} \mathrm{C}$;

- échangeur air/sel de $150 \mathrm{~kW}_{\text {th }}$;

- 2 cuves de stockage de $1 \mathrm{~m}^{3}$;

- asservissement des températures, des débits, et des niveaux permettant une conduite automatique de l'installation:

- 2 pompes verticales immergées d'un débit de 1 et $2,5 \mathrm{~m}^{3} \mathrm{~h}^{-1}$;

- vannes d'isolement et de réglage à commande électrique ou manuelle.
3. Propriétés physiques du H.T.S. - Le tableau II donne quelques propriétés du H.T.S. comparées avec celles d'autres liquides envisageables pour le stockage de chaleur (l'eau sous pression, un liquide organique et le sodium fondu). Le H.T.S. se distingue surtout par sa masse volumique importante et par sa température de fusion élevée $\left(146^{\circ} \mathrm{C}\right)$.

Par contre, il permet d'atteindre des températures de l'ordre de $500^{\circ} \mathrm{C}$ sans se dégrader, alors que les liquides organiques sont limités à $350^{\circ} \mathrm{C}$ et que le stockage de l'eau liquide nécessiterait des réservoirs résistant à des pressions très élevées et serait de toute façon limité vers $300^{\circ} \mathrm{C}$. Seul le sodium permet de couvrir un domaine de température plus étendu que le H.T.S., mais nous verrons plus loin qu'il se prête mal au stockage de chaleur.

Signalons enfin que le H.T.S. est un liquide ionique : les constituants $\mathrm{NaNO}_{2}, \mathrm{KNO}_{3}$ et $\mathrm{NaNO}_{3}$ se dissocient à l'état liquide en cations $\mathrm{Na}^{+}$et $\mathrm{K}^{+}$et en anions $\mathrm{NO}_{2}^{-}$et $\mathrm{NO}_{3}^{-}$. Ces particules chargées électriquement possèdent une certaine mobilité qui rend conducteur électrique le H.T.S.

4. Propriétés thermiques. - 4.1 STOCKage DE CHALEUR. - Le H.T.S. est un excellent fluide de stockage de chaleur. Utilisable depuis $200^{\circ} \mathrm{C}$ jusqu'aux environs de $500^{\circ} \mathrm{C}$, on ne peut guère lui comparer que le sodium. Entre $250^{\circ} \mathrm{C}$ et $450^{\circ} \mathrm{C}$ le H.T.S. peut accumuler sous forme de chaleur $152 \mathrm{kWh} / \mathrm{m}^{3}$ contre

Tableau II. - Propriétés physiques du.H.T.S. et d'autres fluides thermiques. Ces propriétés sont indiquées pour une température de $250^{\circ} \mathrm{C}$, exception faite des températures de fusion et des proprićtés du H.T.S. à $450{ }^{\circ} \mathrm{C}$.

\begin{tabular}{|c|c|c|c|c|c|}
\hline & & $\begin{array}{l}\text { Eutectique } \\
\text { Diphényle- }\end{array}$ & & H.? & \\
\hline & 100 bars & diphényle & Sodium & $250^{\circ} \mathrm{C}$ & $450^{\circ} \mathrm{C}$ \\
\hline & - & - & - & - & - \\
\hline $\begin{array}{l}\text { Masse volumique } \\
\qquad \mathrm{kg} / \mathrm{m}^{3}\end{array}$ & 799 & 871 & 893 & 1895 & 1748 \\
\hline $\begin{array}{l}\text { Chaleur spécifique } \\
\mathrm{kj} / \mathrm{kg} . \mathrm{K}\end{array}$ & 4,84 & 2,56 & 1,32 & $1,56\left(^{*}\right)$ & $1.56\left(^{*}\right)$ \\
\hline $\begin{array}{l}\text { Conductibilité } \\
\text { thermique } \\
\text { W/m.K }\end{array}$ & 0,62 & 0,104 & 78 & $0.43(* *)$ & $0,30(* *)$ \\
\hline $\begin{array}{l}\text { Viscosité } \\
\text { dynamique } \\
\mathrm{N} . \mathrm{S} / \mathrm{m}^{2}\end{array}$ & $1,08 \times 10^{-4}$ & $2.97 \times 10^{-4}$ & $3.92 \times 10^{-4}$ & $4.57 \times 10^{-3}$ & $1.53 \times 10^{-3}$ \\
\hline Nombre de Prandtl & 0,86 & 7,4 & 0,0066 & 15,3 & 7,3 \\
\hline $\begin{array}{l}\text { Température de } \\
\text { fusion }{ }^{\circ} \mathrm{C}\end{array}$ & 0 & 12 & 98 & & 46 \\
\hline
\end{tabular}

(*) Ces valeurs ont été confirmées par des essais menés sous contrat E.D.F., D.E.R. par le laboratoire de physique des liquides ioniques de l'université Paris-VI.

$\left({ }^{* *}\right)$ Les valeurs trouvées dans la littérature pour cette grandeur sont assez disparates; des mesures effectuées en liaison avec le laboratoire de dynamique et thermophysique des fluides de l'Université de Provence ont confirmé la difficulté d'obtenir des résultats reproductibles [4]. 
seulement $61 \mathrm{kWh} / \mathrm{m}^{3}$ pour le sodium avec le même écart de température. Le coût du $\mathrm{kWh}$ stocké est également à l'avantage du H.T.S. (Nous ne comptons que le coût du fluide : environ $2 \mathrm{~F}$ le $\mathrm{kg}$ pour le H.T.S. et $3 \mathrm{~F}$ le $\mathrm{kg}$ pour le sodium.)

4.2 TRANSFERT THERMIQUE. - Le H.T.S. est par contre un fluide de transfert thermique assez médiocre : il permet d'obtenir des coefficients de transfert thermique de 5000 à $8000 \mathrm{~W} / \mathrm{m}^{2} .{ }^{\circ} \mathrm{C}$ si on ne veut pas être trop pénalisé par les pertes de charges dues à une masse volumique importante. Il est donc meilleur que les fluides organiques mais nettement inférieur au sodium et conduit à utiliser des échangeurs de dimensions relativement importantes.

5. La dégradation du H.T.S. - 5.1 EFFETS DE LA DÉGRADATION. - Toutes les propriétés que nous venons de citer sont vraies tant que le mélange de sels fondus conserve sa composition initiale. En pratique on observe souvent les évolutions suivantes :

a) Diminution de la teneur en nitrite, ce qui a pour effet d'augmenter la température de fusion du mélange : la figure 1 montre que cette température de fusion peut alors passer de $146^{\circ} \mathrm{C}$ à plus de $200{ }^{\circ} \mathrm{C}\left({ }^{1}\right)$;

b) formation de carbonates qui provoque également une augmentation de température de fusion;

c) formation d'oxyde de sodium ou de soude, qui sont tous deux des produits très corrosifs.

Il est donc indispensable d'éviter cette dégradation lorsqu'elle risque de se produire.

5. 2 LES RÉACTIONS CHIMIQUES DE DÉGRADATION. Le H.T.S. n'est pas un milieu chimiquement inerte et ses constituants sont susceptibles de réagir entre eux ou avec leur environnement (atmosphère ou parois métalliques). Ces réactions sont complexes et souvent mal connues, mais on peut citer les plus importantes (références [5] et [6]).

La première est une oxydation du nitrite par l'oxygène de l'air en donnant du nitrate :

$$
\mathrm{NaNO}_{2}+\frac{1}{2} \mathrm{O}_{2} \rightleftarrows \mathrm{NaNO}_{3}
$$

La seconde est une réaction de décomposition du nitrite, appelée thermolyse qui produit du nitrate et de l'oxyde de sodium :

$$
5 \mathrm{NaNO}_{2} \rightleftarrows 3 \mathrm{NaNO}_{3}+\mathrm{Na}_{2} \mathrm{O}+\mathrm{N}_{2} \text {. }
$$

(1) Cette courbe expérimentale est en bon accord avec le diagramme de phase du système $\mathrm{NaNO}_{2}-\mathrm{KNO}_{2}-\mathrm{NaNO}_{3}-\mathrm{KNO}_{3}$ [18]. On y retrouve en particulier une rupture de pente à $197^{\circ} \mathrm{C}$ et pour $18 \%$ de nitrite qui correspond sensiblement au début de cristallisation en deux phases distinctes de $\mathrm{NaNO}_{3}$ et $\mathrm{KNO}_{3}$. Une deuxième rupture de pente vers $180^{\circ} \mathrm{C}$ est plus difficilement explicable.

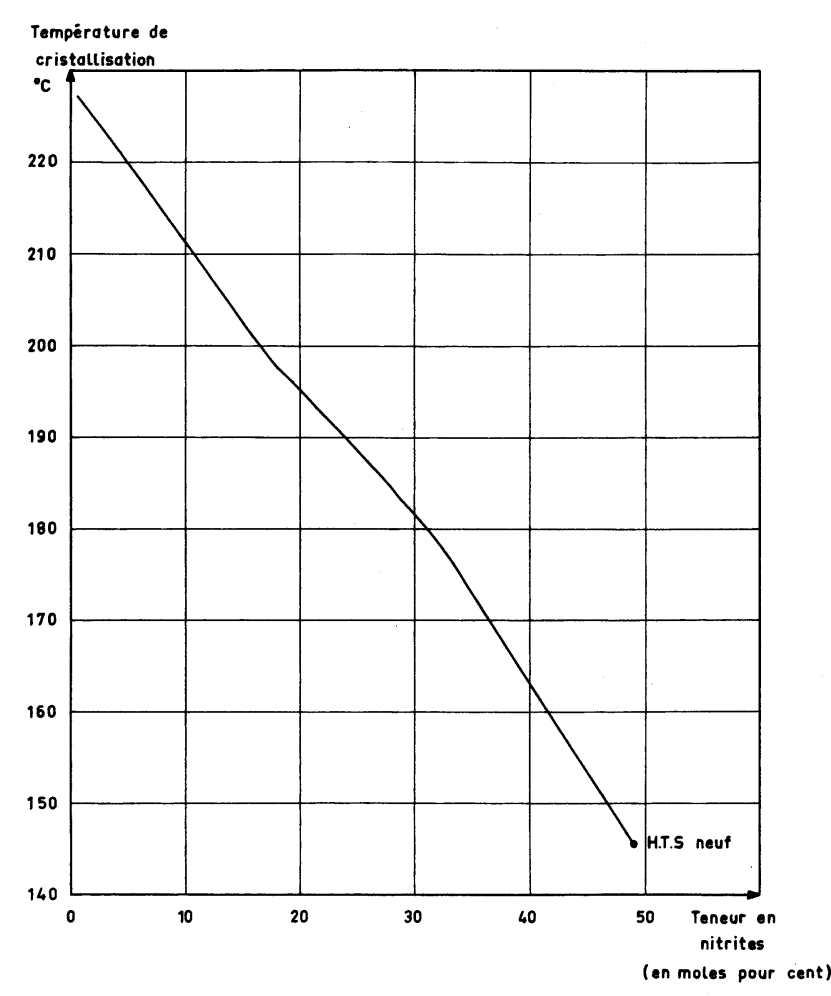

Fig. 1. - Température de cristallisation du H.T.S. dégradé : Lorsque le H.T.S. se dégrade, la teneur en nitrite diminue et la température de cristallisation augmente.

[Crystallization temperature of decomposed H.T.S. : when H.T.S decomposes, nitrite content lessens and crystallization temperature increases.]

L'oxyde de sodium $\mathrm{Na}_{2} \mathrm{O}$ peut alors réagir avec le dioxyde de carbone ou la vapeur d'eau pour former de la soude ou du carbonate de sodium :

$$
\begin{aligned}
& \mathrm{Na}_{2} \mathrm{O}+\mathrm{H}_{2} \mathrm{O} \rightarrow 2 \mathrm{NaOH} \\
& \mathrm{Na}_{2} \mathrm{O}+\mathrm{CO}_{2} \rightarrow \mathrm{Na}_{2} \mathrm{CO}_{3}
\end{aligned}
$$

(Nous avons écrit toutes ces réactions comme si elles ne faisaient intervenir que les sels de sodium; en fait, compte tenu de l'état ionisé elles concernent indifféremment le sodium ou le potassium.)

En simplifiant à l'extrême, on peut supposer que seules les réactions (1) et (2) interviennent de façon notable : elles vont alors amener le mélange à un état d'équilibre que l'on peut calculer $\left({ }^{2}\right)$ et qui est représenté sur les figures 2 et 3 (en ne tenant pas compte des réactions (3) et (4) on néglige les teneurs en $\mathrm{CO}_{2}$ et $\mathrm{H}_{2} \mathrm{O}$ de l'air ou de l'azote de qualité industrielle).

${ }^{2}$ ) Les caractéristiques thermodynamiques des corps considérés sont disponibles dans les tables de thermochimie $[16,17]$ excepté pour $\mathrm{NaNO}_{2}, \mathrm{KNO}_{2}$, et $\mathrm{K}_{2} \mathrm{O}$ liquide. Nous avons supposé faute de renseignement plus précis que l'enthalpie libre de fusion de $\mathrm{K}_{2} \mathrm{O}$ était égale à celle de $\mathrm{Na}_{2} \mathrm{O}$. L'équilibre (1) a été évalué à partir des résultats cités dans les références [7] et [8]. On obtient pour chaque réaction la variation d'enthalpie libre :

$$
\begin{aligned}
& \Delta G_{1}=-25150+23,7 \mathrm{~T} \text { en } \mathrm{kcal} / \mathrm{mole} \\
& \Delta G_{2}=9050+3,9 \mathrm{~T} .
\end{aligned}
$$




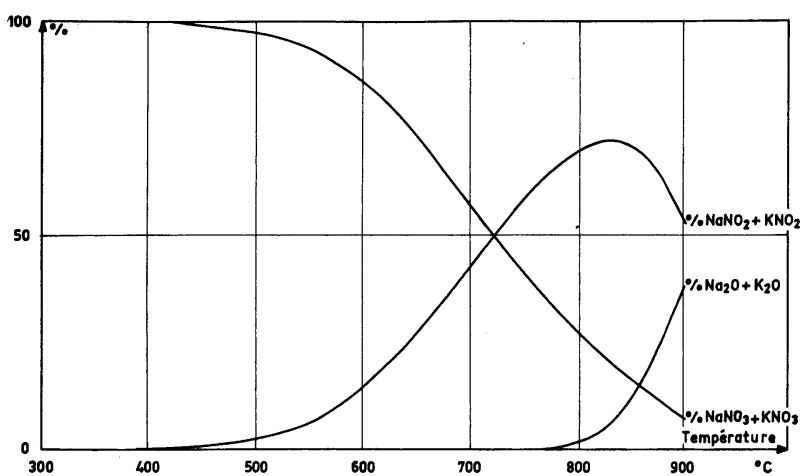

Fig. 2. - Composition théorique du H.T.S. à l'équilibre sous air en moles pour cent.

[Theoretical composition of H.T.S. at the equilibrium under air in mole percent.]

La figure 2 montre que sous air, la teneur en nitrite à l'équilibre est très faible aux températures normales d'utilisation du H.T.S. : le risque de disparition du nitrite par la réaction (1) est grand. Par contre pour des températures supérieures à $600^{\circ} \mathrm{C}$, le nitrite devient relativement stable et la réaction (1) peut même s'inverser avec un dégagement d'oxygène. L'oxyde de sodium n'apparaît de façon importante qu'au-delà de $750^{\circ} \mathrm{C}$.

En présence d'azote et avec une faible pression partielle d'oxygène, on observe un glissement des courbes vers les basses températures (Fig. 3). La teneur en nitrite à l'équilibre augmente sensiblement et passe de $2,6 \%$ sous air à $27,3 \%$ sous azote pour la même température de $500{ }^{\circ} \mathrm{C}$ ce qui est très favorable. Par contre l'oxyde de sodium peut apparaître en quantités importantes dès $600{ }^{\circ} \mathrm{C}$ et il y a un risque de corrosion accru ainsi qu'une possibilité de dégradation suivant la réaction de thermolyse.

Ces courbes théoriques sont bien entendu à considérer avec une certaine réserve. Par exemple, la teneur résiduelle en oxygène de l'azote que nous avons prise égale à un pour mille peut varier de

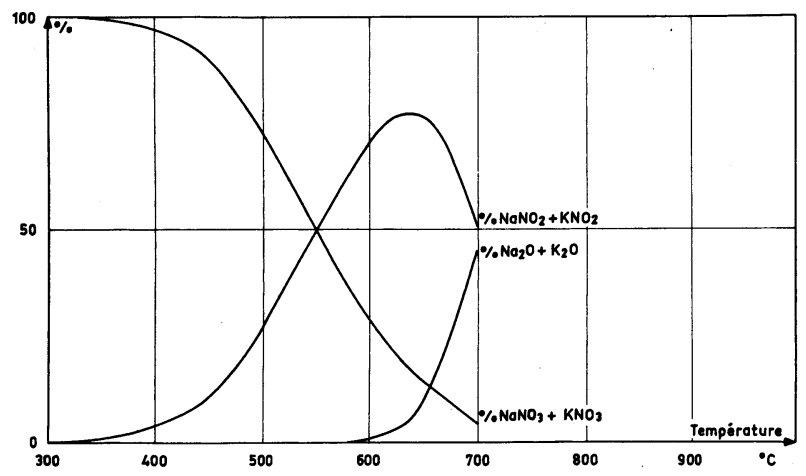

Fig. 3. - Composition théorique du H.T.S. à l'équilibre sous azote pour une pression partielle d'oxygène de $0,001 \mathrm{~atm}$.

[Theoretical composition of H.T.S. at the equilibrium under nitrogen for $0.001 \mathrm{~atm}$. oxygen pressure.] plusieurs ordres de grandeur d'une installation à une autre.

Elles ne montrent pas non plus en combien de temps on atteindra l'état d'équilibre. La cinétique. des réactions chimiques de dégradation est un problème plus ardu.

Elles permettent cependant de mieux comprendre le processus de dégradation du H.T.S. ainsi que les influences du gaz de couverture.

5.3 VITESSE DE DÉGRADATION. - Il reste à savoir en combien de temps on va atteindre l'équilibre, et dans quelle mesure peuvent intervenir les réactions (3) et (4).

La vitesse d'oxydation du nitrite par l'oxygène a été étudiée par E. S. Freeman (référence [7] et [8]) au-dessus de $600{ }^{\circ} \mathrm{C}$. Ces résultats sont donc difficilement utilisables mais on retiendra que la vitesse d'oxydation du nitrite est proportionnelle à la surface de contact entre le sel fondu et l'atmosphère ainsi qu'à la racine carrée de la pression partielle d'oxygène.

Nous avons donc été conduits à faire quelques essais pour mesurer cette vitesse de dégradation $[9,10]$. Sur les figures 4 et 5 le taux de dégradation est repré-

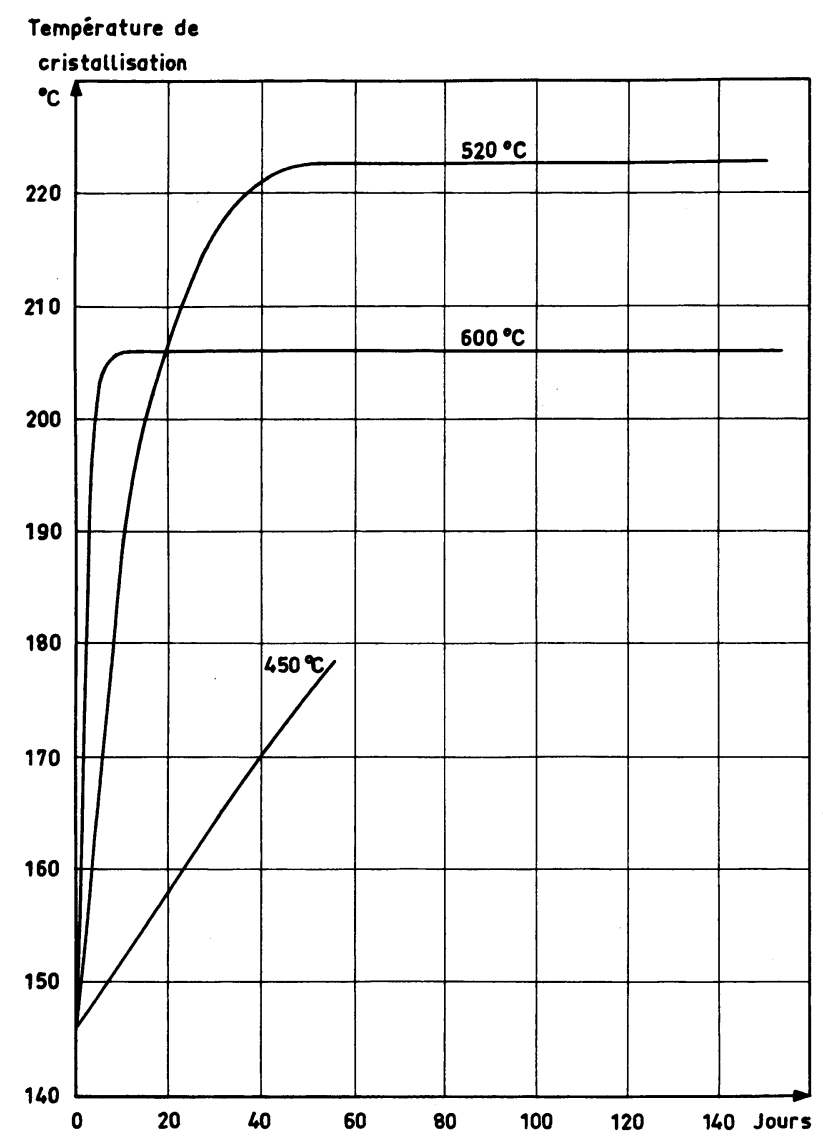

Fig. 4. - Essais de dégradation du H.T.S. à l'air libre : évolution de la température de cristallisation d'un échantillon de sel maintenu à température constante.

[Decomposition test of H.T.S. exposed to the atmosphere : increase of crystallization temperature of a salt sample held at a constant temperature.] 


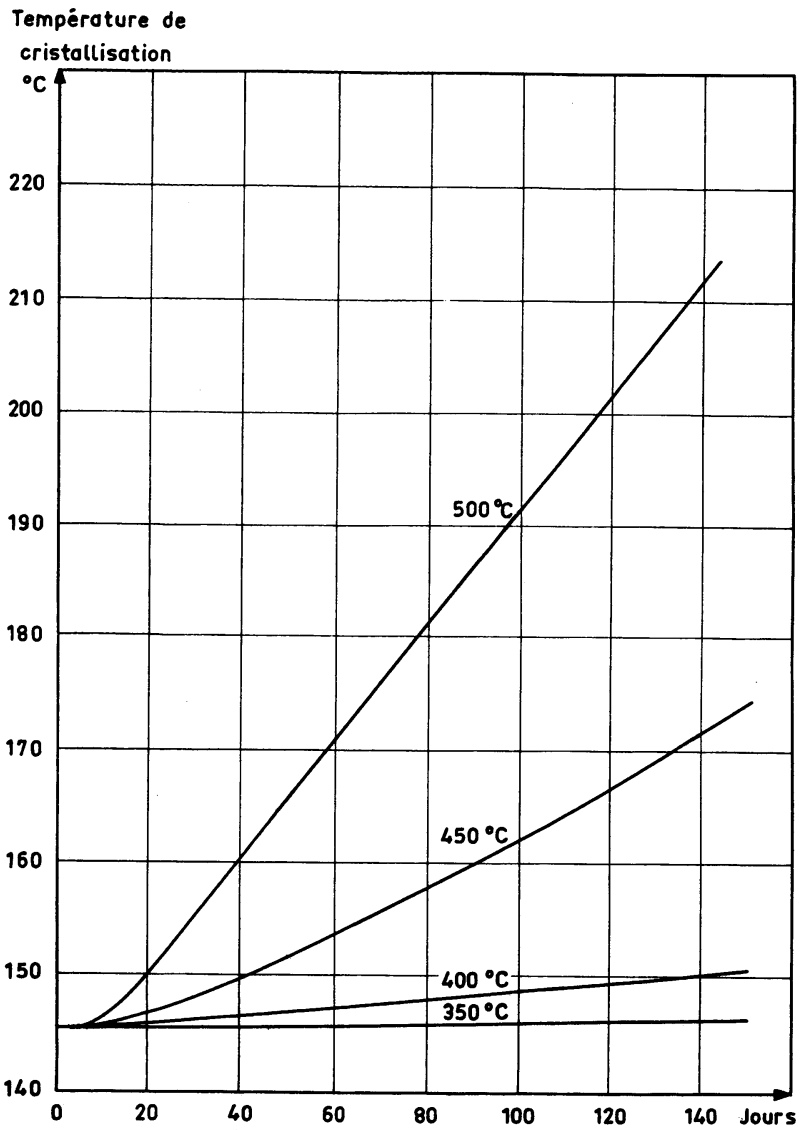

Fig. 5. - Essais de dégradation du H.T.S. en présence d'une petite quantité d'air confiné.

[Decomposition test of H.T.S. under a small quantity of confined air.]

senté par la température de fusion. Trois configurations ont été étudiées :

- sel maintenu à l'air libre (Fig. 4);

- sel en présence d'une petite quantité d'air confiné (Fig. 5);

- sel recouvert d'azote.

5.4 DéGradation sous air [9] et [10]. - Lorsque l'air atmosphérique à accès aux réservoirs de sel fondu, la dégradation du sel se produit principalement par oxydation du nitrite suivant la réaction (1). La figure 2 montre qu'aux températures habituelles d'utilisation du H.T.S. la disparition du nitrite peut être totale.

La vitesse de dégradation fait intervenir plusieurs paramètres :

a) la température : la figure 4 montre l'évolution $\mathrm{du}$ point de fusion du sel maintenu à différentes températures à l'air libre. A $600^{\circ} \mathrm{C}$, la réaction est terminée en quelques jours mais il subsiste une quantité notable de nitrites (environ $13 \%$ ). A $520^{\circ} \mathrm{C}$, la réaction est nettement plus lente mais à la fin il ne reste pratiquement plus de nitrite. $\mathrm{A} 450^{\circ} \mathrm{C}$ la réaction n'est toujours pas terminée après 50 jours ; b) la surface libre du sel en contact avec l'atmosphère : en effet, la dégradation met en jeu des liquides et des gaz et l'interface liquide-gaz en sera donc l'emplacement privilégié ;

c) les mouvements de convection qui peuvent renouveler l'oxygène et le nitrite présents à l'interface. La figure 5 montre que si l'on évite le renouvellement de l'air (essais sous air confiné) la dégradation est sensiblement moins rapide ;

d) les parois du réservoir peuvent exercer un effet catalytique ou inhibiteur non négligeable suivant la nature du métal utilisé (référence [11]).

La réaction de thermolyse peut également intervenir mais elle ne peut produire qu'une très faible quantité de $\mathrm{Na}_{2} \mathrm{O}$, aussi cette réaction ne pourra se poursuivre que si l'oxyde de sodium est détruit par le dioxyde de carbone ou par l'eau suivant les réactions (3) et (4). $\mathrm{La}$ réaction de thermolyse est donc limitée par la faible teneur en $\mathrm{CO}_{2}$ (environ $300 \mathrm{ppm}$ ) et par l'humidité de l'atmosphère.

5.5 DéGradation sous azote [4]. - Les essais de laboratoire n'ont montré aucune dégradation du H.T.S. après 6 mois sous azote à $500^{\circ} \mathrm{C}$. Dans des installations industrielles la dégradation du H.T.S. sous couverture d'azote ne devient gênante qu'au bout de plusieurs années.

Il ne subsiste donc qu'une faible dégradation qui peut se produire aussi bien par oxydation que par thermolyse et les paramètres qui vont fixer la vitesse de dégradation sont alors les suivants.

a) teneur du gaz de couverture en impuretés $\left(\mathrm{H}_{2} \mathrm{O}, \mathrm{CO}_{2}, \mathrm{O}_{2} \ldots\right)$;

b) fréquence de renouvellement de ce gaz;

c) étanchéité du circuit (risques d'introduction d'air dans le circuit d'azote).

Cette dégradation dépend des conditions d'exploitation du circuit à sels fondus, mais elle reste généralement très faible. La protection la plus efficace contre la dégradation du H.T.S. est donc l'azote. Son emploi devient pratiquement indispensable au-dessus de $400{ }^{\circ} \mathrm{C}$.

5.6 PrinCiPe de la RÉGÉNÉRATION du H.T.S. [10]. - On peut dans une large mesure prévenir la dégradation du H.T.S. Il semble cependant exclu de maintenir le mélange à sa composition initiale pendant toute la durée de vie (30 ans) d'une centrale solaire. Il arrivera donc que l'on soit obligé de régénérer le sel parce que sa température de fusion aura augmenté. La teneur en nitrite du sel doit alors être ramenée à sa valeur initiale et il faudra pour cela : - évacuer une partie du sel dégradé, pauvre en nitrite ;

- la remplacer par un mélange plus riche en nitrite.

Ce nouveau mélange devra être riche en nitrite et en plus il ne devra pas modifier les proportions 
de sels de sodium et de sels de potassium; il y a donc deux possibilités :

a) utiliser un mélange de deux nitrites $\mathrm{NaNO}_{2}$ $\mathrm{KNO}_{2}$ qui a l'avantage de réduire au maximum la quantité de sel à évacuer, mais qui utilise un produit coûteux, le nitrite de potassium ;

b) utiliser un mélange $\mathrm{NaNO}_{2}-\mathrm{KNO}_{3}$ nettement moins cher mais en quantités plus importantes.

Le choix entre ces deux solutions dépend des prix des produits et du transport.

Il peut également arriver que des quantités importantes de produits basiques se forment avec risques de corrosion ( $\mathrm{NaOH}$ ou $\mathrm{Na}_{2} \mathrm{O}$ ). On peut alors détruire ces produits par addition d'un sel acide comme le bichromate de potassium $\mathrm{K}_{2} \mathrm{Cr}_{2} \mathrm{O}_{7}$ en faible quantité, ce qui ne modifie pas les propriétés du mélange.

$\mathrm{Si}$ on souhaite réduire les quantités de déchets, à chaque opération de régénération, il serait préférable d'utiliser un mélange plus riche en sels de sodium, par exemple le mélange binaire à $50 \%$ en masse de nitrite de sodium et $50 \%$ de nitrate de potassium dont nous reparlerons plus loin.

6. Corrosion et problèmes de matériaux [9]. - 6.1 MÉTAUX. - Les nitrates et les nitrites étant des sels oxydants, leur principale action sur les aciers est la formation de couches d'oxydes plus ou moins importantes, même si on utilise une couverture d'azote. d'azote.

Les aciers au carbone ou les aciers faiblement alliés sont utilisables sans problème jusqu'à $400^{\circ} \mathrm{C}$. Au-delà de cette température, l'oxydation s'accélère et devient prohibitive à partir de $450^{\circ} \mathrm{C}$.

Les aciers inoxydables austénitiques sont beaucoup moins sensibles à l'oxydation, par contre au-delà de $500^{\circ} \mathrm{C}$ il apparaît sur certains aciers une corrosion intergranulaire (pour les aciers stabilisés au titane en particulier) dont les effets sur la résistance mécanique sont mal connus.

Il sera donc particulièrement important de savoir si cette action du sel sur les aciers ne va pas perturber le comportement mécanique des aciers et en particulier leur résistance à la fatigue.

On sait relativement peu de choses sur l'influence du gaz de couverture. La plupart des essais de corrosion en sel fondu ont été effectués à l'air et on dispose de peu de données sur la corrosion par le H.T.S. sous couverture d'azote.

D'autres métaux tels que le nickel ou le cuivre sont utilisables. Par contre les métaux fortement réducteurs comme le magnésium peuvent donner lieu à des réactions violentes et doivent être exclus.

6.2 MATÉRIAUX ORGANIQUES [12]. - Les matières inflammables sont susceptibles de réagir avec le H.T.S. et peuvent donner lieu à des combustions plus ou moins rapides. Elles doivent donc être évitées. Avant d'utiliser toute matière organique on devra donc s'assurer qu'elle ne présente aucun risque d'inflammation spontanée en présence de H.T.S.
6.7 Autres MAtÉRIAUX. - L'alumine peut être utilisée sans problème au contact du H.T.S. La porcelaine ou la silice sont attaquées rapidement si le sel contient de l'oxyde de sodium ou de la soude.

Nous avons testé des éprouvettes de béton au contact du sel fondu : le béton se fissure sous l'effet de la température et le H.T.S. ne semble pas aggraver ce comportement. A température ambiante, des essais de courte durée ont montré que le H.T.S. dissous dans l'eau n'avait pas d'action sur le béton.

7. Autres mélanges de sels fondus envisageables. Le mélange H.T.S. qui est le plus fréquemment utilisé comme fluide thermique et que nous avons décrit correspond à la composition suivante :

$\begin{array}{rc}\mathrm{NaNO}_{2} & 40 \% \text { en masse } \\ \mathrm{KNO}_{3} & 53 \% \\ \mathrm{NaNO}_{3} & 7 \%\end{array}$

Cependant, dans différentes applications thermiques ou métallurgiques d'autres mélanges obtenus à partir des mêmes produits de base sont utilisés. Leurs températures de fusion sont souvent plus élevées que celle du H.T.S., mais ils peuvent avoir d'autres avantages. On rencontre principalement :

a) Le mélange binaire

$$
\begin{array}{rl}
\mathrm{NaNO}_{2} & 50 \% \text { en masse } \\
\mathrm{KNO}_{3} & 50 \%
\end{array}
$$

qui fond à $149{ }^{\circ} \mathrm{C}$ mais présente l'avantage d'être plus riche en nitrite que l'eutectique, ainsi la dégradation a pour effet de diminuer dans un premier temps la température de fusion, ce qui augmente le délai possible entre deux opérations de régénération. La température de fusion du mélange passe par un minimum lorsque la teneur en $\mathrm{NaNO}_{2}$ est d'environ $40 \%$.

b) Le mélange binaire

$$
\begin{array}{cl}
\mathrm{NaNO}_{3} & 42 \% \text { en masse } \\
\mathrm{KNO}_{3} & 58 \%
\end{array}
$$

qui fond à $225^{\circ} \mathrm{C}$ mais ne contient pas de nitrite et ne nécessite pas de couverture d'azote.

c) On ajoute parfois au H.T.S. une faible teneur en eau qui a pour effet d'abaisser le point de fusion et d'améliorer la conductibilité thermique. Ce procédé est malheureusement inutilisable pour le stockage thermique car la solubilité de l'eau décroît rapidement avec la température et l'eau se vaporise rapidement au-dessus de $250^{\circ} \mathrm{C}$ [13].

8. Technologie [3]. - 8.1 LES RISQUES DE FIGEAGE. - La principale difficulté dans la conception d'une installation à sels fondus vient de la température de fusion élevée de ces produits.

Si la température en un point du circuit s'abaisse en dessous de $146^{\circ} \mathrm{C}$, le sel risque de se solidifier et d'obstruer les canalisations ou de bloquer les 
vannes. Un certain nombre de précautions sont à prendre pour éviter ce problème.

On doit d'abord prévoir un calorifuge efficace et un système de chauffage de secours, ou traçage, que nous décrirons plus loin.

Les points du circuit les plus exposés seront les tuyauteries de faible diamètre qui s'obstruent beaucoup plus vite que les grosses canalisations.

Il faut aussi éviter de laisser le sel immobile dans une canalisation. Si une partie du circuit reste inutilisée pendant plusieurs heures, il est préférable de la vidanger.

Rappelons enfin que le sel solide est plus dense que le liquide : la solidification du sel se fera avec une diminution de volume, par contre la fusion provoque une expansion importante et elle doit être faite avec beaucoup de précaution pour éviter des ruptures de tubes.

8.2 Le traçage. - Nous avons vu que l'ensemble du circuit à sels fondus doit être équipé d'un dispositif de chauffage auxiliaire qui devra éviter tout refroidissement accidentel en dessous du point de fusion du H.T.S. Ce chauffage peut être réalisé de deux façons :

a) le traçage électrique : on dispose le long du circuit à sel fondu des câbles chauffants électriques. Bien que sa réalisation soit plus simple, ce système a l'inconvénient de ne pas assurer une température constante et pour maintenir par exemple $200^{\circ} \mathrm{C}$ en tout point du circuit on peut avoir localement des températures nettement supérieures et une dépense d'énergie surabondante;

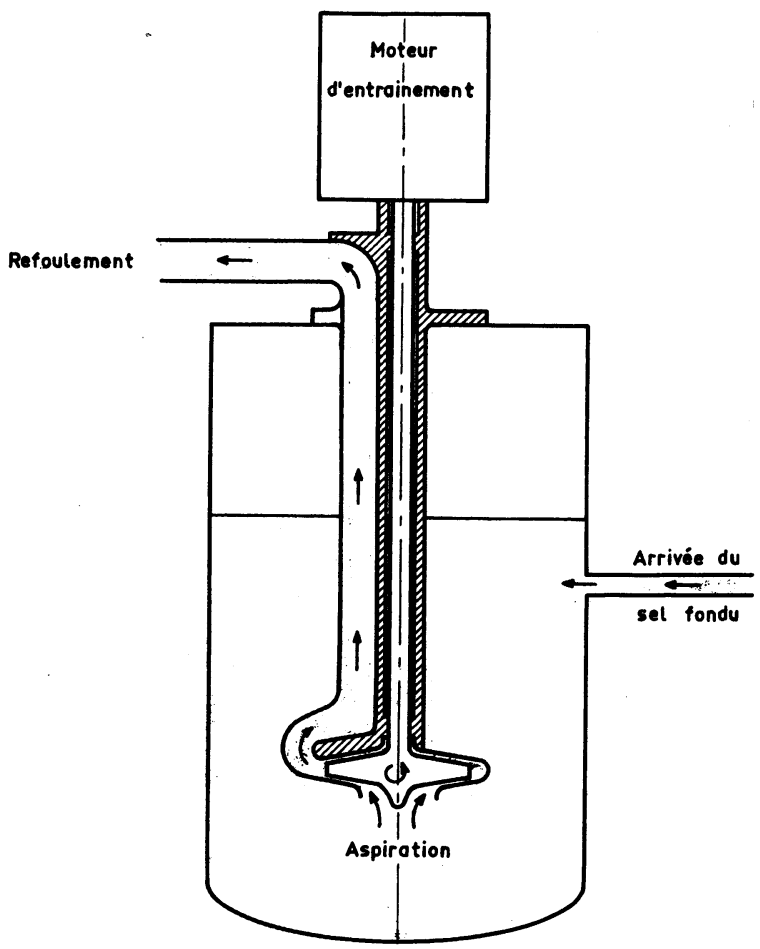

Fig. 6. - Pompe à axe vertical.

[Vertical pumps.] b) le traçage à la vapeur : les canalisations sont constituées de tubes à double paroi. Dans l'espace annulaire circule de la vapeur sous pression et la température ne peut descendre en dessous de la température de condensation. Ce système offre plus de sécurité que le traçage électrique mais coûte plus cher.

8.3 Composants. - Compte tenu des risques de figeage, on voit que le H.T.S. présente une certaine analogie avec le sodium et dans une large mesure, la technologie du sodium peut être reproduite.

Ainsi, comme pour le sodium, on utilisera des pompes à axe vertical afin de ne pas avoir de problèmes d'étanchéité sur des pièces tournantes. La figure 6 montre la disposition d'une telle pompe : elle se trouve placée dans une cuve à niveau libre et l'arbre traverse le plafond de cette cuve, bien au-dessus du niveau du sel fondu, ce qui évite tout risque de fuite vers l'extérieur. La tension de vapeur du H.T.S. est faible et il y a peu de risque de cavitation.

Le même problème se retrouve au niveau des vannes : l'étanchéité est alors assurée par un soufflet métallique extensible qui relie le clapet au corps de la vanne (Fig. 7).

Les échangeurs de chaleur tubulaires sont d'un emploi fréquent : ils doivent être alors entièrement vidangeables et en aucun cas le sel ne doit se solidifier à l'intérieur d'un échangeur.

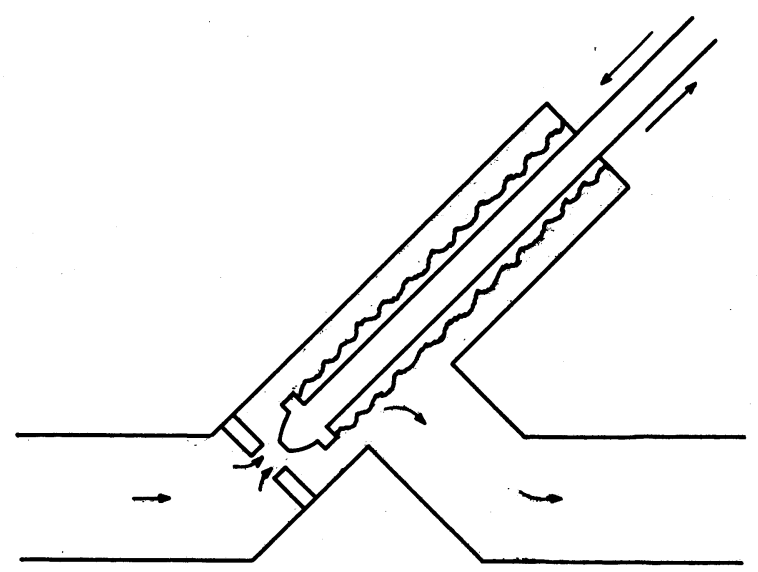

Fig. 7. - Vanne à soufflet.

[Valve with bellows.]

9. Sécurité $[12,14]$. - L'utilisation d'un liquide à haute température et en grande quantité nécessite bien entendu des précautions, quelle que soit la nature de ce liquide.

Il faut noter en particulier que si l'utilisation du H.T.S. comme fluide de transfert thermique peut être considérée comme sûre, il n'en a pas toujours été de même pour les applications métallurgiques.

La règle sans doute la plus importante à observer est d'éviter de maintenir à proximité d'une installation utilisant le H.T.S. tout produit combustible. En effet, si le H.T.S. ne peut pas brûler, il peut faciliter 


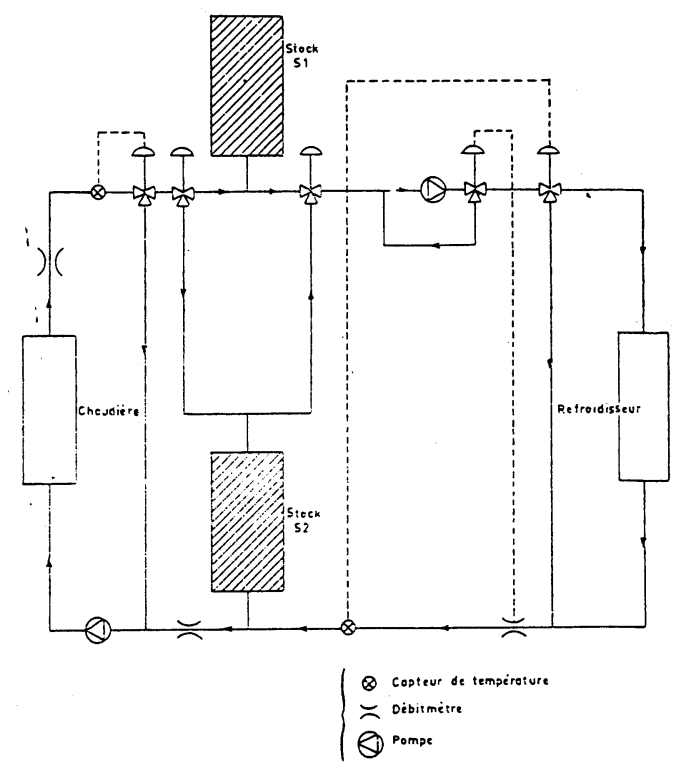

Fig. 8. - Schéma de la boucle à sels fondus.

[Molten Salts test loop.]

la combustion d'autres produits par un apport d'oxygène. Dans certains cas, la combuistion peut être très violente, ou même explosive : ainsi dans des ateliers de traitements thermiques d'alliages légers, des accidents graves se sont produits à la suite de l'introduction d'alliages de magnésium ou de copeaux d'aluminium dans des mélánges fondus de nitrates et de nitrites.

Dans le cas d'une installation de stockage de chaleur ou de transfert thermique, ce risque est heureusement très faible et un contrôle strict des matières utilisées devrait constituer une garantie suffisante sans être une contrainte gênante pour l'exploitation.

Il ne faut pas négliger la toxicité des nitrites ou même des nitrates : ces produits sont utilisés par l'industrie alimentaire mais seulement en faibles quantités. L'absorption de doses plus importantes peut provoquer des troubles plus ou moins graves. Son contact fréquent avec la peau peut aussi provoquer des dermatoses.

Si une surchauffe importante du sel se produit, cela peut engendrer un dégagement d'oxygène accompagné d'azote et de vapeurs nitreuses : ces dernières étant toxiques il est indispensable que l'installation soit suffisamment ventilée.

A la suite des opérations de régénération du sel on peut avoir à se débarrasser de masses de sel dégradé assez importantes : la meilleure solution consiste à les mettre sous une forme permettant de les recycler pour la préparation de nouveaux mélanges de sels fondus. Leur utilisation comme engrais semble possible mais nécessiterait des études plus approfondies $\left({ }^{3}\right)$.

10. Conclusion. - Le H.T.S. est aujourd'hui utilisé par diverses industries depuis près d'un demisiècle. Son utilisation comme fluide de stockage thermique bénéficie de cette expérience mais a nécessité l'approfondissement de certains problèmes : en particulier la dégradation du sel et le comportement des aciers qui sont simultanément soumis à l'action $\mathrm{du}$ sel et aux chocs thermiques dus aux variations d'ensoleillement [15].

En définitive, le H.T.S. est actuellement le meilleur fluide de stockage de la chaleur solaire à haute température car il permet au stockage particulièrement dense pour un faible coût.

( $\left.{ }^{3}\right)$ E.D.F., pour ce problème, a pris des contacts avec l'INRA ; il semble que les nitrites, si les quantités ne sont pas trop importantes, puissent se comporter en engrais; il n'y a néanmoins, pour le moment, aucune certitude.

\section{Bibliographie}

[1] Kirst, W. E., Nagle, W. M., Castner, J. B., Trans. A.I.Ch.E. 36 (1940) 371.

[2] Voznide, H. P., Uhl, V. W., Chem. Eng. (1963) p. 129.

[3] Allard, J. P., Stockage thermique dans les sels fondus. Dimensionnement d'une boule expérimentale. Programme d'essais. Rapport E.D.F. P52/D08/76/34.

[4] Contrat d'Etude E.D.F./Université de Provence. Laboratoire de Dynamique et thermophysique des Fluides.

[5] Alexander, J. Jr., Hindin, S. G., Ind. Eng. Chem. 39 (1947) 1044.

[6] LesCheWSKI, K., Bérichte 72B (1939) 1763.

[7] Freeman, E. S., J. Phys. Chem. 60 (1956) 1487.

[8] Freeman, E. S., J. Phys. Chem. 79 (1957) 838.

[9] Allard, J. P., Roche, M., Sel de stockage H.T.S. Stabilité. Corrosion. Résultats d'essais. Rapport E.D.F. P52/D08/ 78. 25.

[10] Gauger, A., Etude expérimentale de la régénération du H.T.S. et du sel binaire $\mathrm{KNO}_{3} \mathrm{NaNO}_{2}$. Rapport E.D.F. P52/D38/79. 13.
[11] Lalchandani, R., Grover, P. D., Indian Chem. Eng. 14 (1972) 34.

[12] Flamant, G., Nitrites. Nitrates. Toxicité, sécurité appliquées à Thémis. Rapport E.D.F. C.N.R.S. P55 Them 78.68.

[13] Hull, H. S., Turnbull, A. G., J. Phys. Chem. 74 (1970) 1783.

[14] Notice Technique « Hitec». E. I. Du Pont de Nemours and Co (Inc.) Explosive Department. Chemical Products Sales Division, Wilmington Delaware 19898.

[15] Contrat d'études EDF/Ecole Nationale Supérieure de Mécanique et d'Aérotechnique; Laboratoire de Mécanique et de Physique des Matériaux.

[16] JANAF, Thermochemical Tables. Second Edition. Stull, D. R. and PrOPHET, H. et al. NSR DS-NBS 37.

[17] Thermochemical Properties of Inorganic Substances, BARIN and KNACKE (Springer-Verlag) 1963, Berlin.

[18] Phase Diagrams For Ceramists, Levins, E. M., RobBins, C. R., McMurdie, H. F., The American Ceramic Society 1964. 